\title{
June updates: people and places
}

\section{Rs Prizes for 2018}

In March, the NC3Rs in the UK presented Rickie Patani of the Francis Crick Institute and UCL Queen Square Institute of Neurology with the 2018 International 3Rs Prize for his work published in the journal Cell Reports, 'Progressive Motor Neuron Pathology and the Role of Astrocytes in a Human Stem Cell Model of VCP-Related ALS' The paper evaluated patient-derived stem cells as a means to study amyotrophic lateral sclerosis, a fatal degenerative motor neuron disease, without the use of animal models. The main prize comes with a $£ 30 \mathrm{k}$ award, sponsored by GSK.

The NC3Rs also recognized two highly commended papers, with awards of $£ 5 \mathrm{k}$ each. Diogo Mosqueira from the University of Nottingham was recognized for his paper in the European Heart Journal titled 'CRISPR/Cas9 editing in human pluripotent stem cell-cardiomyocytes highlights arrhythmias, hypocontractility, and energy depletion as potential therapeutic targets for hypertrophic cardiomyopathy.' Bernhard Voelkl from the University of Bern was recognized for his PLoS Biology paper titled 'Reproducibility of preclinical animal research improves with heterogeneity of study samples.'

\section{Custom mouse microbiomes at Taconic Taconic Biosciences has announced the launch of a new program, TruBiome, to generate and maintain mice with custom microbiome profiles for its customers. Available profiles include the Wild Mouse gut microbiome (recently licensed from the NIH National Institute of Diabetes and Digestive and Kidney Disease), Altered Schaedler Flora profile, and others. \\ "Decades of experience leading the industry in commercial germ-free mouse production, germ-free derivation, and gnotobiotic husbandry services provide Taconic with a strong foundation to enable customers to access the solutions they need to address microbiome research challenges," commented chief executive office Nancy Sandy in a press release. "The launch of TruBIOME is exciting because it gives researchers the ability to learn how}

microbial variability impacts their animal model experiments."

\section{4 years and counting...}

Charles River Laboratories and the CHDI

Foundation have announced an extension to their ongoing collaboration to develop novel therapeutics for Huntington's Disease (HD), a fatal genetic disorder that causes the progressive breakdown of nerve cells in the brain. The collaboration between the preclinical contract research organization and non-profit dedicated to HD research began in 2005 and will continue for at least five more years.

"Together, Charles River and CHDI have identified novel potential drug targets, bred and validated translational research animal models, run large screens and generated proof-of-concept molecules, and evaluated the potential efficacy of both small molecule and biologic candidates in preclinical models of HD," noted a press release marking the extension.

Charles River has also announced a licensing agreement with CrownBioscience to distribute its Zucker Diabetic SpragueDawley (ZDSD) rat, a proprietary inbred, polygenic rat that develops metabolic syndrome, obesity, dyslipidemia, and diabetes. "We are proud to expand access to the ZDSD model. Metabolic diseases, particularly diabetes, are increasingly common and have significant impact globally. Providing access to a translatable model to study this important set of diseases will help progress research forward, toward the ultimate goal of delivering safe, effective therapeutics to patients," commented Colin Dunn, Corporate Senior Vice President of Global Research Models \& Services at Charles River, in a press release.

\section{NCI-MATCH designation for JAX}

In human-focused news, The Jackson Laboratory's Clinical Genomics Laboratory (CGL) has recently been designed as an National Institutes of Health National Cancer Institute (NCI) Molecular Analysis for Therapy Choice (MATCH) laboratory. As such, the CGL will study tumor gene abnormalities and help clinicians refer patients to potential NCIMATCH clinical precision medicine cancer treatment trials, according to a press release from the Jackson Laboratory.

"As a nonprofit institute, this program allows JAX to continue our unswerving focus on genetics and genomics to significantly improve human health," Honey Reddi, Clinical Lab director at JAX Genomic Medicine, commented in the release. "To play a role in connecting patients-often those with no other options-with precision medicine cancer trials, to help clinicians give their patients better options, is truly a gift."

\section{AAALAC accreditation for EyeCRO} EyeCro, a preclinical contract research organization dedicated to ophthalmic drug discovery and development, has recently been granted AAALAC accreditation for its year-old Oklahoma City, Oklahoma facility, joining four other organizations in the state and about 1000 others worldwide in receiving the endorsement that denotes commitment to animal care.

"Our team is excited to receive this tremendous honor that recognizes our commitment to strong science and the highest standards of preclinical research," commented Alexander Quiambao, Vice President of Operations, in a press release. "This enhances our ability to assist companies around the world which are advancing vision-saving therapies that can treat various forms of blindness."

\section{Oklahoma baboons to move to Texas} The University of Oklahoma will end its pathogen-free olive baboon breeding program, funded by the National Institutes of Health since 1998. Animals from the colony will be transferred this summer to the University of Texas MD Anderson Cancer Center's Michale E. Keeling Center, located near Bastrop, Texas. The decision to end the program at Oklahoma was announced by then-president David Boren in September 2015.

Published online: 21 May 2019 https://doi.org/10.1038/s41684-019-0310-1 